\title{
Needle Stick Injury From a COVID-19 Patient-Fear It or Forget It?
}

\author{
Vishakh C. Keri · Parul Kodan • Anubhav Gupta • \\ Pankaj Jorwal
}

Received: 5 December 2020 / Accepted: 20 January 2021 / Published online: 23 July 2021

(C) Journal of Bioethical Inquiry Pty Ltd. 2021

\section{Dear Editor,}

The ongoing pandemic has thrown many challenges to healthcare workers (HCW's) specially in terms of having safe working conditions and using Personal Protective Equipment (PPE). Inherent to the COVID19 , is its high transmissibility through respiratory droplets, aerosols, and fomites (van Doremalen et al. 2020). As a result, HCW's must wear PPE all the time while caring for COVID-19 patients. Fogging of the goggles and face shields leads to poor vision which puts them at high risk for various injuries including a needle stick injury while performing medical procedures and phlebotomy. Moreover, many HCW's from diverse specialities are being mobilized to care for the overwhelming number of these patients, some of them are not accustomed to performing such procedures routinely leaving them vulnerable to a potential

V. C. Keri $(\square)$

Infectious Diseases, Department of Medicine

and Microbiology, All India Institute of Medical Sciences,

New Delhi, India

e-mail: vckeri@gmail.com

P. Kodan $\cdot$ P. Jorwal

Department of Medicine, All India Institute of Medical

Sciences, New Delhi, India

e-mail: parulkodan@yahoo.com

\section{A. Gupta}

Department of Transfusion Medicine, ABVIMS \& RML

Hospital, New Delhi, India health hazard. Coupled with this comes the fear and anxiety of having got infected with COVID-19 by or immediately after a needle stick injury.

Published studies shed little light on viremia due to SARS CoV-2, and the available literature reports rates between 2.8 per cent to 15 per cent (Kim et al. 2020; Huang et al. 2020). However these reported intermittent viremia in blood. Data on SARS patients has shown that the viral load in plasma is low and no transfusion transmission has been demonstrated. Studies that screened blood donations for SARSCoV RNA in 2003 did not identify any positives (Chang, Yan, and Wang 2020). American Association of Blood Banks (AABB) does not make any recommendation on deferral of blood donation by recovered COVID-19 patients or their close contacts in view of inadequate data suggesting transfusion related transmission (American Association of Blood Banks 2020). While National AIDS Control Organisation (NACO) suggests deferring blood donation 28 days post exposure for contacts and confirmed cases (National Blood Transfusion Council, Ministry of Health and Family Welfare, Government of India 2020). Although there appears to be a theoretical risk of transmission of infection through blood, it is still extremely low due to low volume of blood in needle stick injuries compared to the well-known respiratory route. A published case report in which an aplastic anaemia patient had received apheresis platelet transfusion from a person who later turned out to be COVID-19 also did not demonstrate any 
transfusion related COVID-19 infection (Cho et al. 2020). Hence, the fear over transmission of infection by needle stick injury seems unwarranted.

While the literature is still evolving, it is prudent to follow standard precautions and safe needle handling practises to avoid needle stick injuries. This is more important than ever because although risk of getting COVID-19 is low, the risk of getting HIV, Hepatitis $\mathrm{B}$, and Hepatitis $\mathrm{C}$ infection and other transfusion transmitted infections remains quite real and pragmatic. If a HCW happens to accidentally prick himself, he or she should follow the standard post-exposure prophylaxis guidelines for HIV and Hepatitis B and get to know the sero-status of the involved patient as early as possible. All HCW's should get themselves vaccinated with Hepatitis B vaccine and have their antibody titres checked regularly. Application of soap solution, hand sanitizers, antifogging agents, and iodophors on goggles and face shields leads to decreased incidences of fogging and thus could prevent such injuries by improving visibility ( $\mathrm{Hu}$ et al. 2020). Avoidance of panic and hurry would also go a long way in preventing such episodes in a healthcare setting.

\section{Declarations}

Declaration of Conflict of Interest The authors declare no conflict of interest.

\section{References}

American Association of Blood Banks. 2020. Update: Impact of 2019 novel coronavirus and blood safety [Internet]. http://www.aabb.org/advocacy/regulatorygovernment/ Documents/Impact-of-2019-Novel-Coronavirus-onBlood-Donation.pdf. Accessed June 30, 2021.

Chang, L,. Y. Yan, and L. Wang. 2020. Coronavirus disease 2019: Coronaviruses and blood safety. Transfusion Medicine Review 34(2): 75-80.

Cho, H.J., J.W. Koo, S.K. Roh, et al. 2020. COVID-19 transmission and blood transfusion: A case report. Journal of Infection and Public Health 13(11): 1678-1679.

Hu, Y., L. Wang, S. Hu, and F. Fang. 2020. Prevention of fogging of protective eyewear for medical staff during the COVID-19 pandemic. Journal of Emergency Nursing 46(5): 564-566.

Huang, C., Y. Wang, X. Li, et al. 2020. Clinical features of patients infected with 2019 novel coronavirus in Wuhan, China. The Lancet 395(10223): 497-506.

Kim, J-M., H.M. Kim, E.J. Lee, et al. 2020. Detection and Isolation of SARS-CoV-2 in serum, urine, and stool specimens of COVID-19 patients from the Republic of Korea. Osong Public Health and Research Perspectives 11(3): 112-117.

National Blood Transfusion Council, Ministry of Health and Family Welfare, Government of India. 2020. NBTC Interim guidance for blood transfusion services in view of COVID-19 [Internet]. http://naco.gov.in/sites/default/ files/NBTC\%20GUIDANCE\%20FOR\%20COVID-19.pdf. Accessed June 30, 2021.

van Doremalen, N., T. Bushmaker, D.H. Morris, et al. 2020. Aerosol and surface stability of SARS-CoV-2 as compared with SARS-CoV-1. New England Journal of Medicine 382(16): 1564-1567.

Publisher's Note Springer Nature remains neutral with regard to jurisdictional claims in published maps and institutional affiliations. 